\title{
$A b$ initio calculation of rarefied flows of helium-neon mixture: classical vs quantum scatterings
}

\author{
Lianhua $\mathrm{Zhu}^{\mathrm{a}, *}$, Lei $\mathrm{Wu}^{\mathrm{a}}$, Yonghao Zhang ${ }^{\mathrm{a}}$, Felix Sharipov ${ }^{\mathrm{b}}$ \\ a James Weir Fluids Laboratory, Department of Mechanical and Aerospace Engineering, University of Strathclyde, Glasgow \\ G1 1 XJ, UK \\ ${ }^{b}$ Departamento de Física, Universidade Federal do Paraná, Curitiba, 81531-980, Brazil
}

\begin{abstract}
In order to faithfully simulate rarefied gas flows of light-weight molecules at cryogenic temperatures down to several kelvins, the Boltzmann equation with the differential cross section calculated from the realistic intermolecular potential should be applied. In the present work, the direct simulation Monte Carlo (DSMC) method with ab initio intermolecular potentials is first implemented into the open-source software dsmcFoam + for the simulation of general rarefied gas flows. Then, Fourier and Couette flows of the helium-neon mixture are studied for the temperature ranging from $10 \mathrm{~K}$ to $2000 \mathrm{~K}$, where the differential cross sections calculated from both classical and quantum mechanics have been used. Our simulation results show that the quantum scattering effects on the heat flux and shear stress are non-negligible when the equilibrium temperature is lower than $500 \mathrm{~K}$. Also, for the Fourier flow, the mole fraction distributions calculated from the quantum scattering are significantly different from those of classical scattering.
\end{abstract}

Keywords: Quantum scattering, Heat transfer, Direct simulation Monte Carlo, ab initio potential

\section{Introduction}

Rarefied gas mixtures at low temperature appear in many technological applications such as cryogenic pumps, cryogenic separation/capture of gases [1-3], the cryogenic system used in the ITER project [4] as well as high temperature superconducting (HTS) power devices [5, 6]. Only under the rarefied condition, the flow media in these applications can be maintained in the gaseous phase at cryogenic temperatures. In addition, the vacuum condition is indispensable for the thermal insulation of these low-temperature equipment and systems.

High-fidelity numerical studies of rarefied gas flows in these applications are crucial for the optimal engineering design and operation of the systems [7, 8]. The prevailing numerical method for rarefied gas

\footnotetext{
* Corresponding author

Email addresses: 1.zhu@strath.ac.uk (Lianhua Zhu), lei.wu.100@strath.ac.uk (Lei Wu), yonghao.zhang@strath.ac.uk (Yonghao Zhang)
} 
flows is the direct simulation Monte Carlo (DSMC) technique 9 11. In the collision procedure of the DSMC method, the molecular interaction is handled by phenomenological models with experimentally fitted adjustable parameters such as the hard-sphere (HS), variable-hard-sphere (VHS) and variable soft-sphere (VSS) models 9]. These parameters are usually fitted in a narrow temperature range; thus they can not accurate recovery the correct viscosity at extremely low or high temperatures. While the ab initio potentials available in the literature for all the noble gases and their mixtures (see e.g. Refs. 12 15]) provide an adjustable parameter-free approach for the molecular interactions. They have been successfully applied in the DSMC method by a simple efficient technique [16 18. In particular, DSMC based on ab initio potentials with quantum-scattering [19] has been also developed to handle the quantum effects in the low-weight gas molecular collisions e.g., ${ }^{3} \mathrm{He},{ }^{4} \mathrm{He}$, hydrogen, and neon $20-22$. It is found the quantum effects are nonnegligible for the transport of single-component light-weight gases when the temperature is lower than 300 K.

The aim of this work is to further evaluate the quantum effects on the transport of the binary mixtures of low-weight gases by considering the Fourier and Couette flows of helium (He)-neon ( $\mathrm{Ne}$ ) mixture in the transition regime. The Fourier and Couette flows are both classical benchmark problems in rarefied gas dynamics and have been studied extensively, especially for single-component rarefied gas 23 30. While for the mixed gas case, the corresponding numerical studies were available only recently. Most of these studies use binary-mixture kinetic models [31 39] such as the McCormack model [40, 37] and the Kosuge model [41. There are also a few DSMC studies using HS model 42 and ab initio based DSMC with classical inter-atomic interaction [43. In particular, these studies reveal that in the Fourier flow of mixtures, the mole fraction distribution is strongly affected by the molecular collision models. More recently, there is an experimental measurement of the heat flux [44]. To the best of our knowledge, in all of the previous studies, the gas temperature is around the ambient temperature or higher. Thus, the current study uses the DSMC method based on ab initio potentials with the quantum approach to provide benchmark data over the temperature range from several kelvins to several thousand kelvins. It should be noted that the quantum effects are considered only in intermolecular interactions. The quantum effects in the gas-surface interations [45] are not considered in this study.

\section{Statement of the problems}

Consider a binary-mixture of monatomic gases confined between two vertically placed parallel plates with infinite length and width. The two plates are located at $x=-H / 2$ and $x=H / 2$. The mean (or equilibrium) mole fraction of the mixture between the plates is given as

$$
C_{0}=n_{01} / n_{0}, \quad n_{0}=n_{01}+n_{02},
$$


where $n_{01}$ and $n_{02}$ are the equilibrium number densities of the lighter gas He and heavier one, Ne, respectively; $n_{0}$ is the total number densities of the gas mixtures. The temperature difference in the flow field causes a thermal diffusion in the gas mixtures, leading to a spatial inhomogeneous distribution of mole fraction in the gas at the steady state. Hereinafter, the local mole fraction calculated via the local number densities $n_{1}(x)$ and $n_{2}(x)$ is denoted by $C(x)$.

The solution of the problem in question depends strongly on the rarefaction parameter, defined as

$$
\delta=\frac{H p_{0}}{\mu_{0} v_{0}}
$$

where $p_{0}$ is the equilibrium pressure of the gas mixture, $\mu_{0}$ is its viscosity at the equilibrium temperature $T_{0}$, and $v_{0}$ is the characteristic speed of the mixture molecules, defined as

$$
v_{0}=\sqrt{2 k_{B} T_{0} / m}
$$

with $k_{B}$ being the Boltzmann constant and $m$ being the average atomic mass related to the atomic masses of the two species $m_{1}$ and $m_{2}$ by $m=C_{0} m_{1}+\left(1-C_{0}\right) m_{2}$.

In the Fourier flow, the plates are fixed and their surfaces are maintained at temperatures of $T_{C}$ and $T_{H}$, respectively, with $T_{C}<T_{H}$. The reference temperature $T_{0}$ is chosen as the mean temperature $\left(T_{C}+T_{H}\right) / 2$. In the Couette flow, both plates are kept at the equilibrium temperature $T_{0}$, but the left and right plates move with constant velocities of $U_{w} / 2$ and $-U_{w} / 2$, respectively, in the vertical direction.

Rarefied gas flows are described by the one-particle velocity distribution functions (VDF) $f_{\alpha}\left(t, \boldsymbol{r}, \boldsymbol{v}_{\alpha}\right.$ ), which obeys the Boltzmann equation,

$$
\frac{\partial f_{\alpha}}{\partial t}+\boldsymbol{v}_{\alpha} \cdot \frac{\partial f_{\alpha}}{\partial \boldsymbol{r}}=\sum_{\beta=1}^{2} Q_{\alpha \beta}\left(f_{\alpha}, f_{\beta}\right) \quad(\alpha=1,2),
$$

where $Q_{\alpha, \beta}\left(f_{\alpha}, f_{\beta}\right)$ is the collision operator between the component $\alpha$ and $\beta$, expressed as:

$$
Q_{\alpha \beta}\left(f_{\alpha}, f_{\beta}\right)=\int_{\mathbb{R}^{3}} \int_{\mathbb{S}^{2}} g \sigma_{\alpha \beta}(\chi, g)\left[f_{\beta}\left(\boldsymbol{v}_{\beta}^{\prime}\right) f_{\alpha}\left(\boldsymbol{v}_{\alpha}^{\prime}\right)-f_{\beta}\left(\boldsymbol{v}_{\beta}\right) f_{\alpha}\left(\boldsymbol{v}_{\alpha}\right)\right] d \Omega d \boldsymbol{v}_{\beta} .
$$

Here $\boldsymbol{v}_{\alpha}$ and $\boldsymbol{v}_{\beta}$ are the velocities of the $\alpha$ and $\beta$ types of particles before the collision, $\boldsymbol{v}_{\alpha}^{\prime}$ and $\boldsymbol{v}_{\beta}^{\prime}$ are their post collision velocities, $g=\left|\boldsymbol{v}_{\alpha}-\boldsymbol{v}_{\beta}\right|$ is the magnitude of the relative velocity, $\Omega$ is the unit vector on the unit sphere surface $\mathbb{S}^{2}$ pointing to the direction of post-collision relative velocity, and $\sigma_{\alpha \beta}(\chi, g)$ is the differential cross section (DCS). It is a function of $g$ and the deflection angle of the relative velocity $\chi$ $(0 \leq \chi \leq \pi)$ due to the collision. The DCS can be calculated, either by the quantum or classical mechanics, for a given inter-atomic potential.

The total cross section (TCS) is the integral of $\sigma$ on $\mathbb{S}^{2}$,

$$
\sigma_{T, \alpha \beta}=\int_{\mathbb{S}^{2}} \sigma_{\alpha \beta}(\chi, g) d \Omega .
$$


When the differential cross-section is calculated by the classical mechanics, the TCS will be infinite. Therefore, in the DSMC simulation, a cut-off of the impact parameter $b_{c u t}$ will be used. Details will be given in the next section.

The macroscopic quantities of each species can be calculated from their distribution functions, such as the number density and mean velocity,

$$
n_{\alpha}(\boldsymbol{r})=\int f_{\alpha}\left(\boldsymbol{r}, \boldsymbol{v}_{\alpha}\right) d \boldsymbol{v}_{\alpha}, \quad u_{i \alpha}(\boldsymbol{r})=\frac{1}{n_{\alpha}} \int v_{i \alpha} f_{\alpha}\left(\boldsymbol{r}, \boldsymbol{v}_{\alpha}\right) d \boldsymbol{v}_{\alpha}, \quad i=x, y, z
$$

The mass density of species $\alpha$ is

$$
\rho_{\alpha}=m_{\alpha} n_{\alpha}
$$

The mass density and hydrodynamics velocity of the mixture are given as

$$
\rho=\rho_{1}+\rho_{2}, \quad u_{i}=\left(\rho_{1} u_{i 1}+\rho_{2} u_{i 2}\right) / \rho, \quad i=x, y, z .
$$

Other macroscopic properties of the mixture that are of interest to this article include the temperature

$$
T(\boldsymbol{r})=\frac{1}{3 n k_{\mathrm{B}}} \sum_{\alpha=1}^{2} m_{\alpha} \int V_{\alpha}^{2} f_{\alpha}\left(\boldsymbol{r}, \boldsymbol{v}_{\alpha}\right) \mathrm{d} \boldsymbol{v}_{\alpha},
$$

the heat flux

$$
q_{i}(\boldsymbol{r})=\frac{1}{2} \sum_{\alpha=1}^{2} m_{\alpha} \int V_{\alpha}^{2} V_{i \alpha} f_{\alpha}\left(\boldsymbol{r}, \boldsymbol{v}_{\alpha}\right) \mathrm{d} \boldsymbol{v}_{\alpha}, \quad i=x, y, z,
$$

and the pressure tensor

$$
P_{i j}(\boldsymbol{r})=\sum_{\alpha=1}^{2} m_{\alpha} \int V_{i \alpha} V_{j \alpha} f_{\alpha}\left(\boldsymbol{r}, \boldsymbol{v}_{\alpha}\right) \mathrm{d} \boldsymbol{v}_{\alpha}, \quad i, j=x, y, z,
$$

where $\boldsymbol{V}_{\alpha}=\boldsymbol{v}_{\alpha}-\boldsymbol{u}_{\alpha}$ is the peculiar velocity. In particular, the longitude heat flux and shear stress presented in non-dimensional forms are

$$
Q=-q_{x} T_{0} /\left(p_{0} v_{0} \Delta T\right), \quad \Pi=-P_{x y} v_{0} /\left(p_{0} U_{w}\right)
$$

where $\Delta T=T_{H}-T_{C}$. The quantities $Q$ and $\Pi$ are spatially homogeneous in the Fourier and Couette flows, respectively.

The gas-surface interaction is assumed to be fully diffuse which means the particles hitting the solid wall are reflected with a Maxwellian distribution. For example, at $x=-H / 2$, the VDF of the reflected molecules for component $\alpha$ is

$$
\left.f_{\alpha}\right|_{v_{x}>0}=\frac{n_{w, \alpha}}{\left(2 \pi R_{\alpha} T_{w}\right)^{3 / 2}} \exp \left[-\frac{v_{x}^{2}+\left(v_{y}-U_{w} / 2\right)^{2}+v_{z}^{2}}{2 R_{\alpha} T_{w}}\right],
$$

where $n_{w, \alpha}=-\sqrt{2 \pi / R_{\alpha} T_{w}} \int_{v_{x}<0} v_{x} f_{\alpha} d \boldsymbol{v}, R_{\alpha}=k_{B} / m_{\alpha}$. 


\section{The DSMC Method}

The DSMC method provides the same solution as that obtained by solving the Boltzmann equation [46]. In the DSMC method, the movement and collision of gas molecules are simulated by artificial Lagrangian particles with each particle representing $F_{N}$ real molecules, where $F_{N}$ is an adjustable parameter used to balance the precision and computational cost. The computational domain is divided into cells that are used for choosing potential collision pairs and sampling the macro variables. In a DSMC evolution step, each simulated particle undergoes firstly a free-flying step with its current velocity. Then, in each cell, a certain number of particle pairs are chosen as candidate collision pairs. Following the no-time-counter (NTC) method [9, 10, the number of candidate collision pairs are

$$
N_{\text {coll }}=N_{\alpha}\left(N_{\alpha}-1\right) F_{N}\left(\sigma_{T, \alpha \alpha} g\right)_{\max } \Delta t /\left(2 V_{c}\right), \quad \alpha=1 \text { or } 2,
$$

for particles of the same component $\alpha$ and

$$
N_{\text {coll }}=N_{\alpha} N_{\beta} F_{N}\left(\sigma_{T, \alpha \beta} g\right)_{\max } \Delta t /\left(V_{c}\right), \quad \alpha \neq \beta
$$

for particles of different components, where $N_{\alpha}$ and $N_{\beta}$ are the numbers of simulated particles for the component $\alpha$ and $\beta$, respectively; $V_{c}$ is the cell volume; $\Delta t$ is the time step, and $\left(\sigma_{T, \alpha \beta} g\right)_{\max }$ is the maximum of the product of the TCS and $g$. In the current dsmcFoam+ solver, the NTC method is used with the sub-cell technique to promote near-neighbour collisions [47]. Each chosen pair of particles from the $N_{\text {coll }}$ potential pairs execute the collision only if they satisfies the condition

$$
\frac{\sigma_{T} g}{\left(\sigma_{T} g\right)_{\max }}>R_{f}
$$

where $R_{f}$ is a random number uniformly varying from 0 to 1 . The velocities of the particles selected for collision are changed according to the deflection angle $\chi$ and the impact angle $\epsilon$ [9, 11. The impact angle $\epsilon$ is randomly chosen from $[0,2 \pi]$. The deflection angle $\chi$ is calculated from the DCS that depends on the relative velocity $g$ and the interaction potential.

\subsection{Deflection angle and total cross-section}

The DCS is calculated according to both quantum [19, 20] and classical mechanics [17, 48]. Due to the complexity of calculating the DCS from the $a b$ initio potentials, the deflection angles for a series of discrete relative velocities $g_{i}$ have been pre-calculated as two-dimensional matrices for each type of collision pairs (He-He, He-Ne, and Ne-Ne) with both classical and quantum scattering. The values of the relative speed $g_{i}$ are distributed as $g_{i}=G\left(1.005^{j}-1\right)$, where $G=400 \mathrm{~m} / \mathrm{s}$ for the He-He and He-Ne interactions, while $G=200 \mathrm{~m} / \mathrm{s}$ for the Ne-Ne interactions. Each row of the matrices lists the equally probable deflection angles for each $g_{i}$. The total number of discrete relative velocities is 900 for all matrices, and their range is around $2 \mathrm{~m} / \mathrm{s}$ to $35.2 \mathrm{~km} / \mathrm{s}$ for He-He and He-Ne collisions, and $1 \mathrm{~m} / \mathrm{s}$ to $17.6 \mathrm{~km} / \mathrm{s}$ for Ne-Ne collisions. The 
matrices of deflection angle with quantum scattering for the He and Ne self-collisions used in this study are from the Supplementary Material ("xiHe4.csv" and "xiNe.csv") of Ref. 22. We have calculated the matrix of deflection angle for the He-Ne collision using the method described in Ref. [20] based on the potential obtained in Ref. [15. The matrix is provided in the Supplementary Material ("xiHe4-Ne.csv") of the present article. To test the generated matrix, the viscosity and heat conductivity of the He-Ne mixture have been calculated by the technique described in Ref. [17. It has been verified that these transport coefficients obtained by the DSMC method are in agreement within $0.1 \%$ difference with those reported in Ref. [19] over the temperature range considered in the present paper. The matrices of deflection angle with classical scattering have been calculated by the method described in Ref. 17] and have the same discrete relative velocities $g_{i}$ as in the quantum ones.

For the quantum scattering, the TCS-values are also pre-calculated for all the chosen relative velocities and are provided along with the $\chi$-lookup tables. They are plotted as functions of $g$ in Fig. 1, which shows that the variations of TCS with $g$ are similar for the three collision pairs when $g$ is relatively high. All of them decrease slowly with the increase of $g$. However, at low $g$, the variations of the TCS with $g$ are completely different. The TCS of the He-He collision shows a sharp decrease with the increase of $g$ and then ( $g>130 \mathrm{~m} / \mathrm{s}$ ) behaves like a damping oscillation as $g$ increases further. The TCS of the Ne-Ne collision at low $g$ is quite complex and unpredictable. The TCS of the He-Ne collision at low $g$ slightly increases with the increase of $g$.

While in classical-scattering based on ab initio potentials, the range of the molecular interaction is infinite; thus we assume the particles have no interaction if the impact distance is larger than a specific value $d_{\max }$, leading to a constant TCS of $\pi d_{\max }^{2}$. We choose $d_{\max }$ to be $3 r_{0}$ where $r_{0}$ is the inter-atomic distance when their potential equals to zero [17].

The above described technique based on ab initio potentials is implemented as a user-defined collision sub-model in the dsmcFoam+ solver [47] provided in the Supplementary Material. In the implementation, the matrices files are read storing the first hundred columns in the matrix $\xi_{i j}$; the 101st column contains the TCS measured in $\AA^{2}$ for each relative speed $g_{j}$ given in the 102nd column. If a pair has the relative speed $g$, then the index $j$ is calculated by

$$
j=\left\lfloor\frac{\ln (1+g / G)}{\ln 1.005}+\frac{1}{2}\right\rfloor, \quad 1 \leq j \leq 900
$$

and the condition 16 is verified. If the pair is selected for collision, the index $i$ is randomly chosen from the range $1 \leq i \leq 100$. Then, the value $\xi_{i j}$ is used as $\cos \chi$ to calculate new velocities of each molecule using Eqs. (8.32)-(8.35) from Ref. [11].

\subsection{Numerical scheme}

The numerical scheme of the DSMC simulations is the same as that used in Ref. [18, 43]. The domain is decomposed uniformly into 400 cells, and 100 particles are used in each cell on average. The time step 

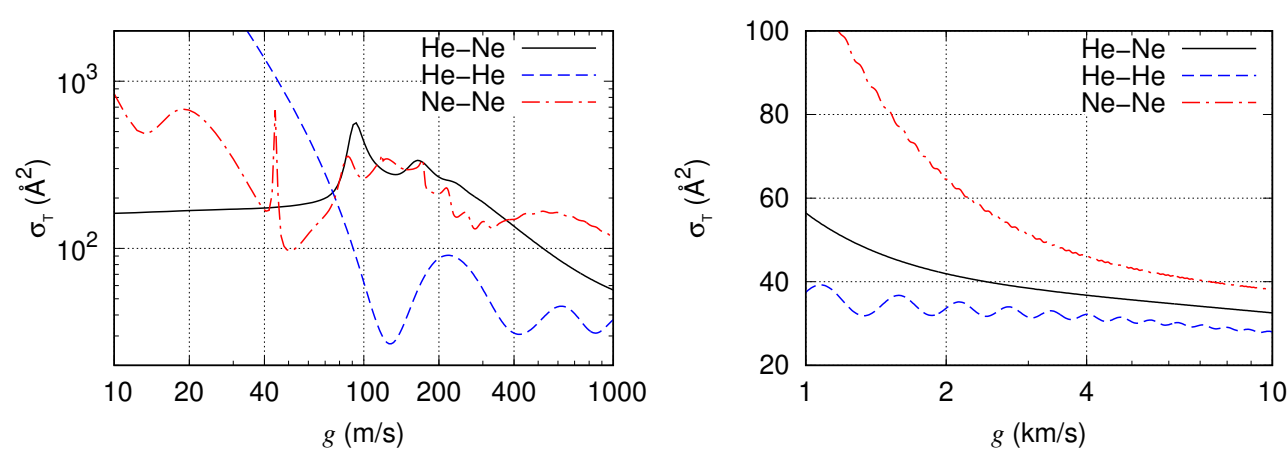

Figure 1: Total cross section $\sigma_{T}$ vs. relative speed $g$ in the scattering using quantum approach.

is $\Delta t=0.002 H / v_{0}$. The simulations start with the particles initialized according to the equilibrium states and run for 100,000 steps, allowing the flow fields to reach steady-state. Then they continue for further 2,000,000 steps for sampling the macro variables including the heat flux and shear stress. The heat flux and shear stress on the plate surface are calculated by accumulating the kinetic energy/momentum changes of the particles striking the wall. Such parameters provide the numerical error less than $0.5 \%$ according to Ref. [18, 43]. Using two 12-core E5-2697 v2 (Ivy Bridge) series CPUs to run the dsmcFoam+ solver, each simulation takes slightly more than two hours. We note that directly solving the Boltzmann equation with the $a b$ initio potentials, e.g., with the fast spectral method [49, 30, the cost may be reduced and the results will be free from statistical noises.

\section{Results and analyses}

\subsection{Fourier flow}

In the Fourier flow, the temperature of the left and right plates are $T_{H}=1.5 T_{0}$ and $T_{C}=0.5 T_{0}$, respectively. The flow is simulated for all the combinations of the following values of the rarefaction parameter $\delta=$ $0.1,1,10$, and of the mole fraction $C_{0}=0.1,0.5,0.9,0.95$. Several values of the equilibrium temperature $T_{0}$ in the range from $10 \mathrm{~K}$ to $2000 \mathrm{~K}$ have been considered. Moreover, nine values of the rarefaction parameter $\delta$ in the range from 0.1 to 50 have been considered for $T_{0}=10 \mathrm{~K}$ and $20 \mathrm{~K}$. The viscosities of the mixture for different $C_{0}$ and $T_{0}$ are listed in Table 1.

\subsubsection{Heat flux}

The variations of the non-dimensional heat flux $(Q)$ with the equilibrium temperature $T_{0}$ obtained by both the classical (CL) and quantum (QU) approaches to the modeling of the inter-atomic interactions are shown in Fig. 2 for each combination of $\delta$ and $C_{0}$. The numerical values in the figures are also provided in Table 1 of the Supplementary Material. Several observations from these data are discussed in detail below.

Firstly, when $T_{0}>200 \mathrm{~K}$, the difference in the predicted $Q$ by the classical and quantum approaches is less than $0.57 \%$, among all the $T_{0}, \delta$ and $C_{0}$. In other words, the quantum effects are negligible for the heat 
Table 1: Viscosity of the He-Ne mixture vs. temperatures and mole fraction. The values for $C_{0}=0.1,0.5$, and 0.9 are extracted from Ref. [19]. The values for $C_{0}=0.95$ have been calculated in the present work using the method of Ref [19].

\begin{tabular}{lrrrr}
\hline$T_{0}(\mathrm{~K})$ & \multicolumn{4}{c}{$\mu_{0}(\mathrm{\mu Pa} \cdot \mathrm{s})$} \\
\cline { 2 - 5 } & $C_{0}=0.1$ & $C_{0}=0.5$ & $C_{0}=0.9$ & $C_{0}=0.95$ \\
\hline 10 & 1.77765 & 1.81960 & 1.99499 & 2.04258 \\
20 & 3.21303 & 3.42891 & 3.45582 & 3.41511 \\
50 & 7.85231 & 7.72304 & 6.67061 & 6.40163 \\
100 & 14.2718 & 13.2567 & 10.6652 & 10.1411 \\
200 & 23.7825 & 21.5907 & 16.9660 & 16.0937 \\
300 & 31.3887 & 28.4216 & 22.3137 & 21.1707 \\
500 & 44.1817 & 40.1113 & 31.6750 & 30.0863 \\
1000 & 70.2338 & 64.3423 & 51.5637 & 49.0983 \\
2000 & 112.544 & 104.396 & 85.3434 & 81.5320 \\
\hline
\end{tabular}

transfer through the He-Ne mixtures when $T_{0}>200 \mathrm{~K}$. We also note that the variation of heat flux with the temperature is not significant when $T_{0}>200 \mathrm{~K}$.

Secondly, as $T_{0}$ decreases from $200 \mathrm{~K}$ to $10 \mathrm{~K}$, the variations of $Q$ with $T_{0}$ calculated by the classical and quantum approaches behave quite differently. On one hand, for the He-dominated mixture $\left(C_{0}=0.9\right.$ or 0.95 ), the $Q$ predicted by the classical approach decreases significantly as $T_{0}$ decreases. For example, in case of $C_{0}=0.95, \delta=10$, the quantity $Q$ decreases from 0.1390 to 0.1129 as $T_{0}$ varies from $200 \mathrm{~K}$ to $10 \mathrm{~K}$. While for $C_{0}=0.1$ and 0.5 , the $Q$ firstly decreases slightly and then increases with a decrease of $T_{0}$. The contrast between these trends is due to the stronger quantum effects in the collision of the lighter-weight He molecules. When He is dominant, the neglect of the quantum effects leads to a much stronger variation of the heat flux with respect to the temperature. This behavior is also observed in the comparative study of single-component gas heat transfer using the two approaches [20. On the other hand, the $Q$ predicted by the quantum approach shows a much smaller variation as $T_{0}$ decreases to $10 \mathrm{~K}$ regardless of $\delta$ and $C_{0}$. The $Q$ increases slightly in most cases, except for the cases with $C_{0}=0.5$ when $T_{0}<20 \mathrm{~K}$, where it shows a significant decrease as $T_{0}$ decreases to $10 \mathrm{~K}$.

Thirdly, regarding the effect of mole fraction on $Q$, we note that the He- or Ne-dominated $\left(C_{0}=0.1\right.$ or $0.95)$ mixtures have significantly lower heat flux values than the mixtures with $C_{0}=0.5$ and 0.9 . It means that the mixing of gases enhances the non-dimensional heat transfer.

Lastly, we observed that the quantity $Q$ decreases quickly as $\delta$ increases from 0.1 to 10 . This trend is more clearly shown in Fig. 3, where the relation between $Q$ and $\delta$ relations with different $C_{0}$ and $T_{0}$ is plotted. We also note that the difference in $Q$ predicted by the classical and quantum approaches is relatively more significant for intermediate $\delta$. This is because, in both the free molecular and continuum limits, the details of the collision models are not important. 

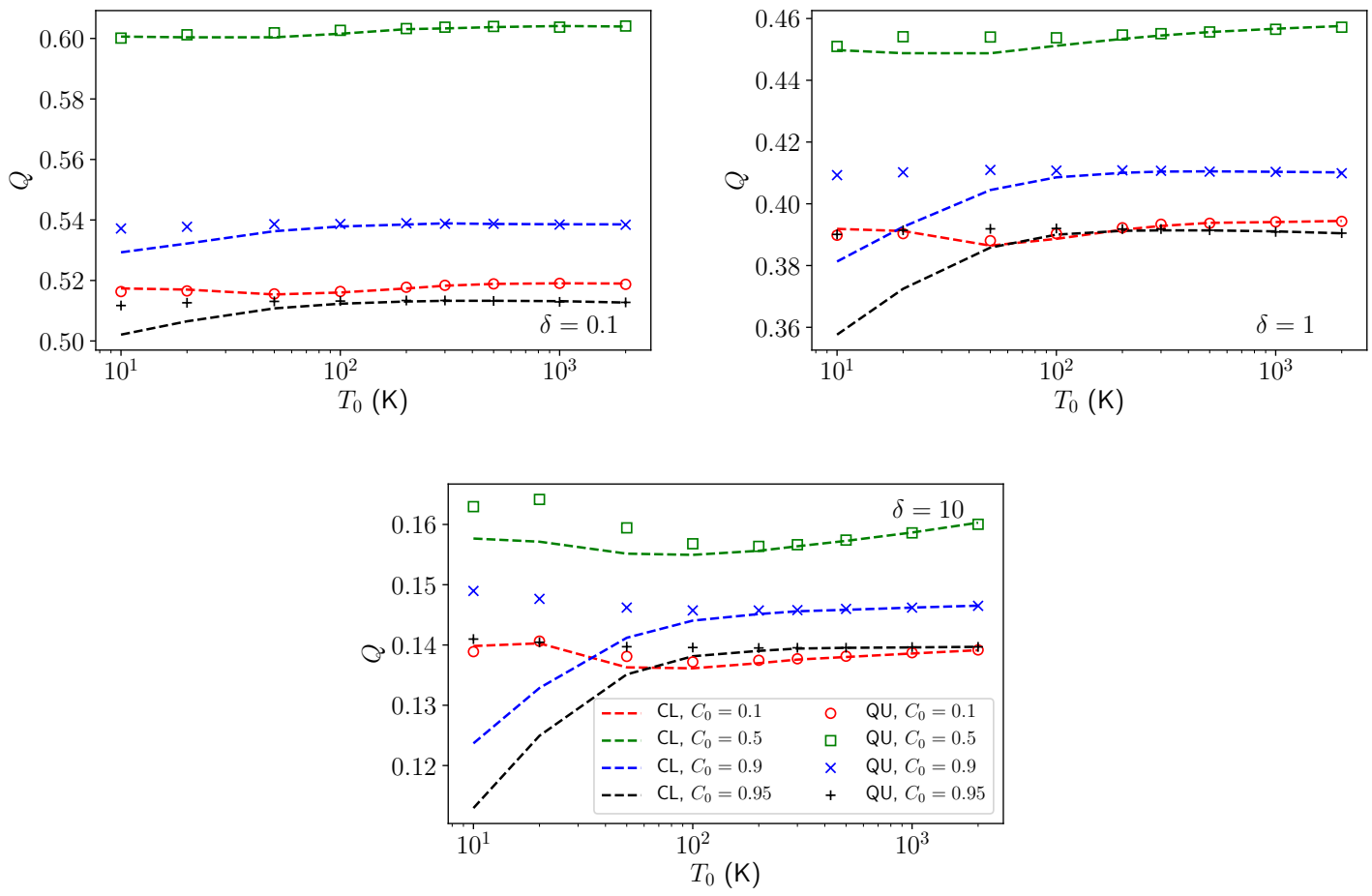

Figure 2: The non-dimensional heat flux $(Q)$ as function of the equilibrium temperature $\left(T_{0}\right)$ predicted by the classical $(\mathrm{CL})$ and quantum (QU) approaches at different rarefaction parameters $(\delta)$ and equilibrium mole fractions $\left(C_{0}\right)$. In each subfigure, the red, green, blue and black lines/symbols represent the results of $C_{0}=0.1,0.5,0.9$, and 0.95 , respectively (color online), in which the lines and symbols correspond to the classical and quantum approaches. The data in theses plots are provided in Table 1 of the Supplementary Material.

\subsubsection{Temperature distribution}

For the currently considered Fourier flow with large temperature difference, the non-dimensional temperature $\left(T / T_{0}\right)$ profile is only weakly affected by the equilibrium temperature $\left(T_{0}\right)$, mole fraction $\left(C_{0}\right)$ and inter-atomic potential model. Here we consider only the effects of inter-atomic potential model and rarefaction parameter. The temperature profiles obtained with several different $T_{0}$ and $C_{0}$ are shown in Fig. 4 . It is seen that the differences between the classical- and quantum-scattering approaches are only observable for the He-dominated mixtures when $T_{0}<50 \mathrm{~K}$. The temperature jumps on the plate surface for $\delta=0.1$ cases are so large that the gas temperatures are below the equilibrium temperatures in the whole domain.

\subsubsection{Mole fraction distribution}

Due to the thermal diffusion, the mole fraction is inhomogeneous for non-isothermal flows. For a stationary mixture flow in the viscous limit $(\delta \rightarrow \infty)$, the temperature gradient leads to a mole fraction gradient as

$$
\frac{\mathrm{d} C}{\mathrm{~d} x}=-\frac{k_{T}}{T} \frac{\mathrm{d} T}{\mathrm{~d} x}
$$



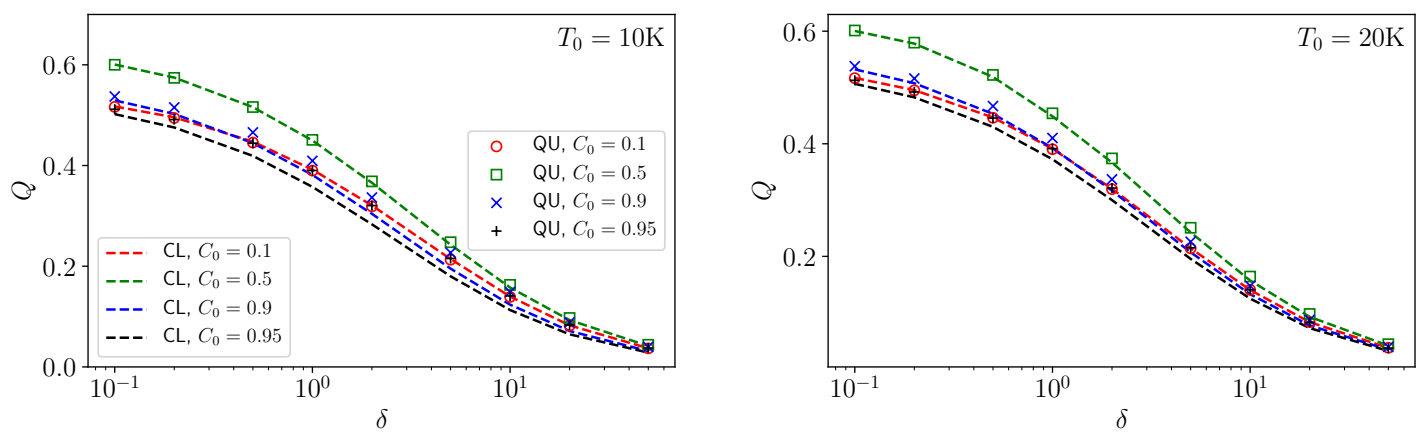

Figure 3: The non-dimensional heat flux $(Q)$ as function of $\delta$ for different $C_{0}$ at $T_{0}=10 \mathrm{~K}$ and $20 \mathrm{~K}$. In each subfigure, the red, green, blue and magenta lines represent the results of $C_{0}=0.1,0.5,0.9$, and 0.95 , respectively (color online), in which the solid and dashed lines correspond to the quantum and classical approaches. The data in this plot are provided in Table 3 of the Supplementary Material.
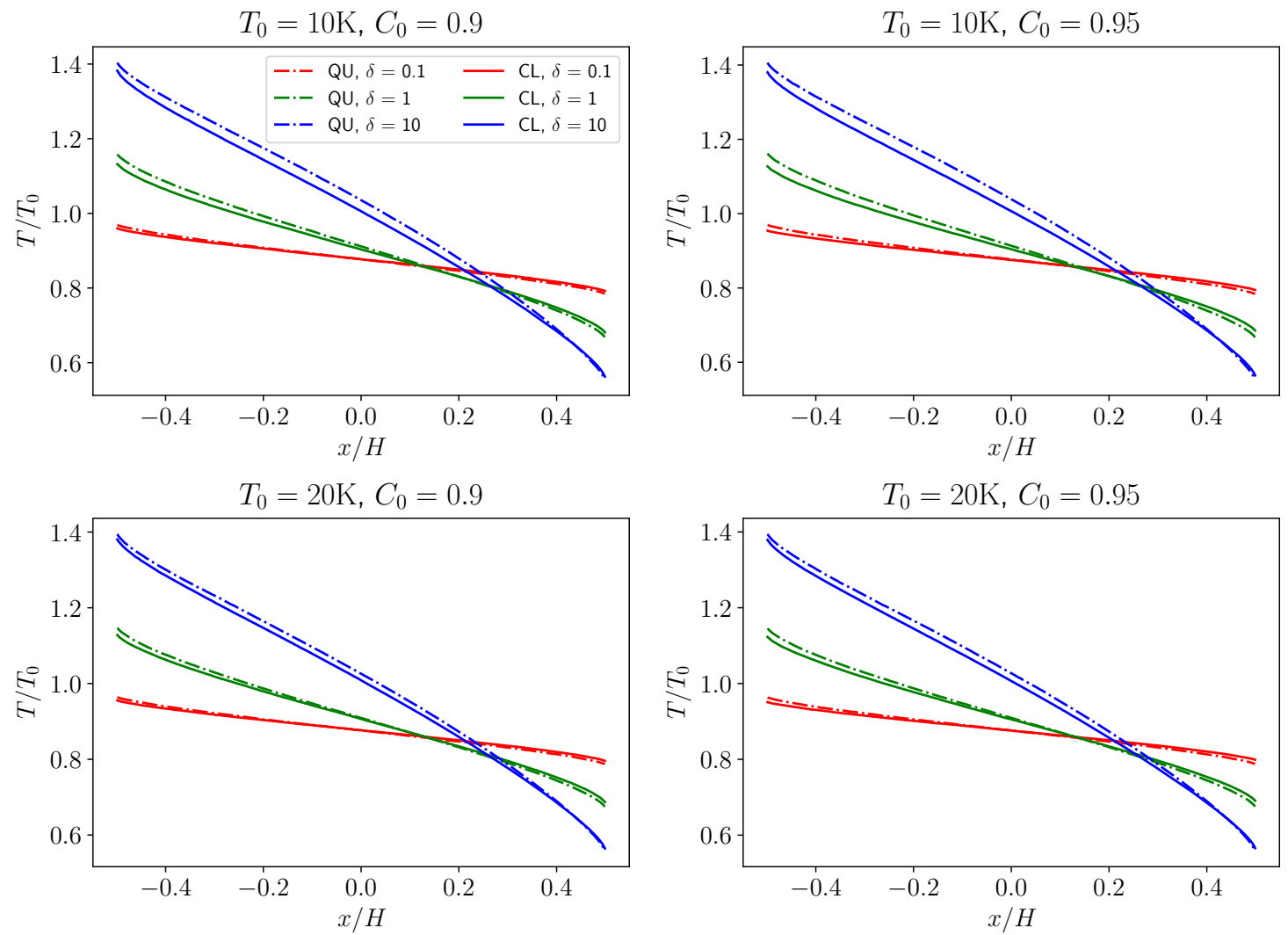

Figure 4: Non-dimensional temperature profiles at different $T_{0}$ and $C_{0}$ for the Fourier flow. In each subfigure, the red, green and blue lines are profiles with $\delta=0.1,1$ and 10, respectively (colorful online). The solid and dashed lines correspond to the quantum- and classical-scattering approaches, respectively. 

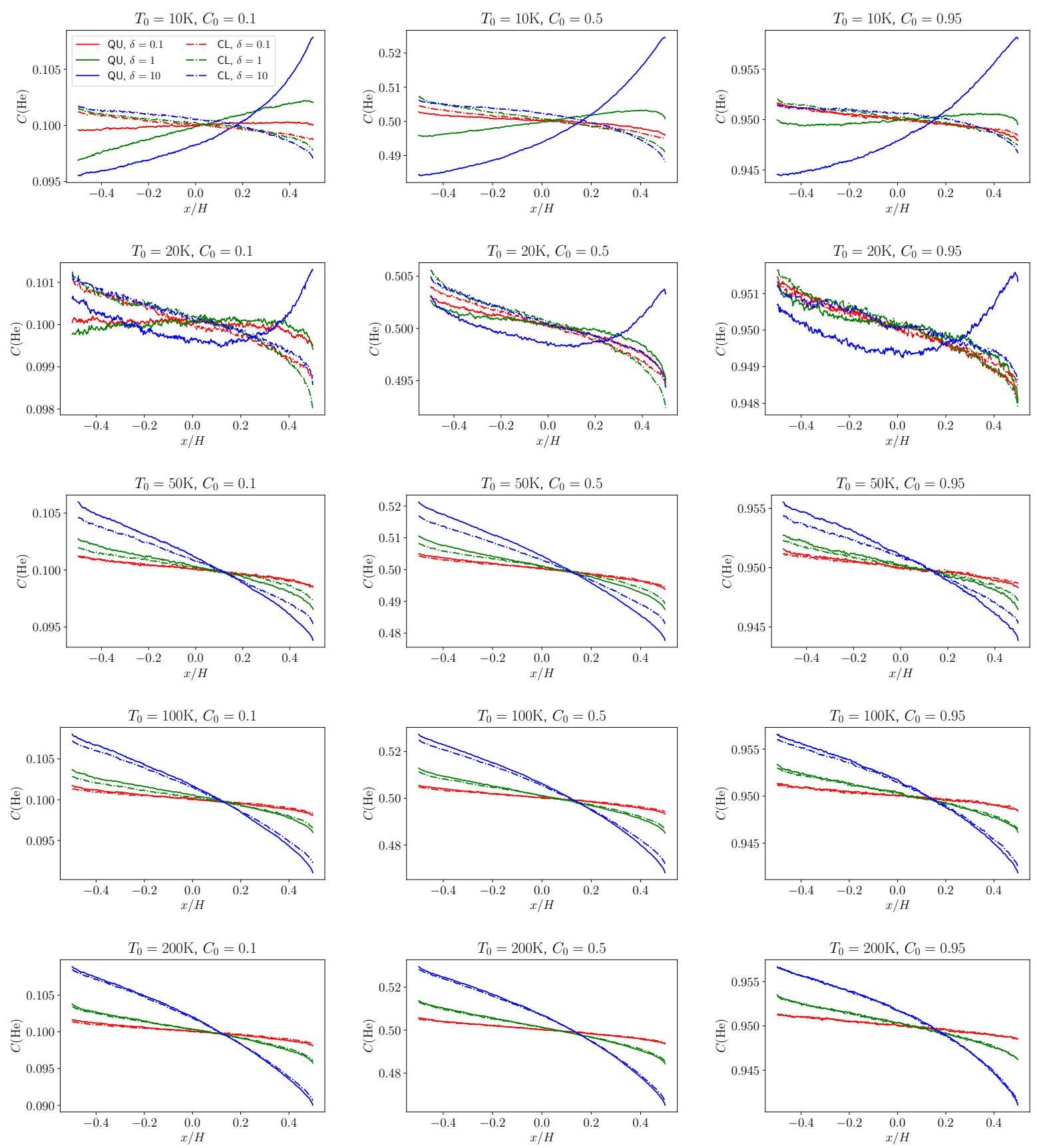

Figure 5: Mole fraction profiles of He at different $T_{0}$ and $C_{0}$ for the Fourier Flow. The line legends are the same as in Fig. 4 
where $k_{T}$ is the thermal diffusion ratio and depends on the local temperature and mole fraction. The thermal diffusion is balanced by the molecular diffusion such that the non-uniform mole fraction is maintained at a steady state. For a gas mixture at a non-cryogenic temperature, its thermal diffusion ratio is negative, meaning that the mole fraction decreases from the high-temperature region to the low-temperature region. However, the reverse of the thermal diffusion ratio sign has been observed for gas mixtures at cryogenic temperatures both experimentally [50, and theoretically based on the Lennard-Jones $(12,6)$ model [50] and the ab initio potential models [19].

The mole fraction profiles for the cases with $T_{0} \leq 200 \mathrm{~K}$ and $C_{0}=0.1,0.5$ and 0.95 are shown in Fig. 5 . The profiles for higher temperatures are not shown due to the negligible difference between the classical and quantum approaches. Figure 5 shows that as $T_{0}$ decreases from $200 \mathrm{~K}$, the difference in the two approaches becomes more significant, which is consistent with the trends of the non-dimensional heat flux presented in Fig. 3. In all the cases, the classical approach always predicts a decreasing mole fraction profiles towards the cold plate, regardless of $T_{0}, C_{0}$ and $\delta$.

On the contrary, the $C$ profiles predicted by the quantum approach have very complex dependence on both $C_{0}$ and $\delta$ when $T_{0} \leq 20 \mathrm{~K}$. When $T_{0}=20 \mathrm{~K}$ and $\delta=10$, the mole fraction predicted by the quantum approach is non-monotonic and has a minimum value between $x=0.1 \mathrm{H}$ and $x=0.15 \mathrm{H}$, which indicates the reverse of the thermal diffusion ratio sign. It happens when the temperature is around 17.9 to $18.6 \mathrm{~K}$, which is indeed very close to the sign-reversing temperature $(18.7 \mathrm{~K})$ of the He-Ne thermal diffusion ratio calculated by the quantum-scattering using $a b$ initio potential [19]. The much stronger fluctuations of $C$ at $T_{0}=20 \mathrm{~K}$ in Fig. 5 , resulting from the much smaller deviations of $C$ from $C_{0}$, also indicates the thermal diffusion ratio is close to zero and is about to change its sign. However, it should be noted that the thermal diffusion ratios in Ref. [19] are obtained under the near equilibrium condition. Meanwhile, for the highly rarefied flows $(\delta=0.1,1)$ with large temperature variation $\left(\Delta T / T_{0}=1\right)$ considered here, these data cannot explain the observed complex behaviors. For example, the $C$ profiles in the case of $T_{0}=20 \mathrm{~K}, C_{0}=0.1$, $\delta=0.1$ and 1 are slightly non-monotonic: they increase slightly first and then decreases towards the cold plate which is opposite to the trend in the case of $\delta=10$. For $T_{0}=10 \mathrm{~K}$ and $\delta=10$ cases, the $C$ profiles change completely to monotonically increasing towards to the cold plate. While for the other $\delta$ cases, the variations are different and depend on $C_{0}$ and $\delta$.

\subsection{Couette flow}

In the Couette flow, the calculations have been carried out for the relative plate velocity $U_{w}$ equal to the characteristic molecular speed $v_{0}$, i.e. $U_{w}=v_{0}$. We consider exactly the same sets of parameters as in the Fourier flow, including $C_{0}, T_{0}$ and $\delta$. 


\subsubsection{Shear stress}

Figure 6 presents the variations of the non-dimensional shear stress with the equilibrium temperature for different $C_{0}$ and $\delta$. The values of the shear stress are also provided in Table 3 of the Supplementary Material. The overall trends of the variations are very similar to those of non-dimensional heat flux in the Fourier flow case. For the He-dominated mixtures, the quantity $\Pi$ calculated by the classical approach decreases clearly as $T_{0}$ decreases from $200 \mathrm{~K}$ to $10 \mathrm{~K}$, while the quantum predictions have only slight variations in the whole temperature range. The maximum relative difference between the two approaches (28\%) appears, as expected, in the case of $T_{0}=10 \mathrm{~K}$ and $C_{0}=0.95$, where the quantum effects are the strongest. For the Ne-dominated mixtures $\left(C_{0}=0.1\right)$, the differences between the two approaches are quite small. In addition, when $T_{0} \geq 200 \mathrm{~K}$, the differences are less than $0.34 \%$. The variations of $\Pi$ with $\delta$ are shown in Figure 7 from which we can see the $\Pi$ drops quickly as $\delta$ increases. Figure 7 also reveals that the differences between the classical and quantum predictions which are more significant in the intermediate values of $\delta$.
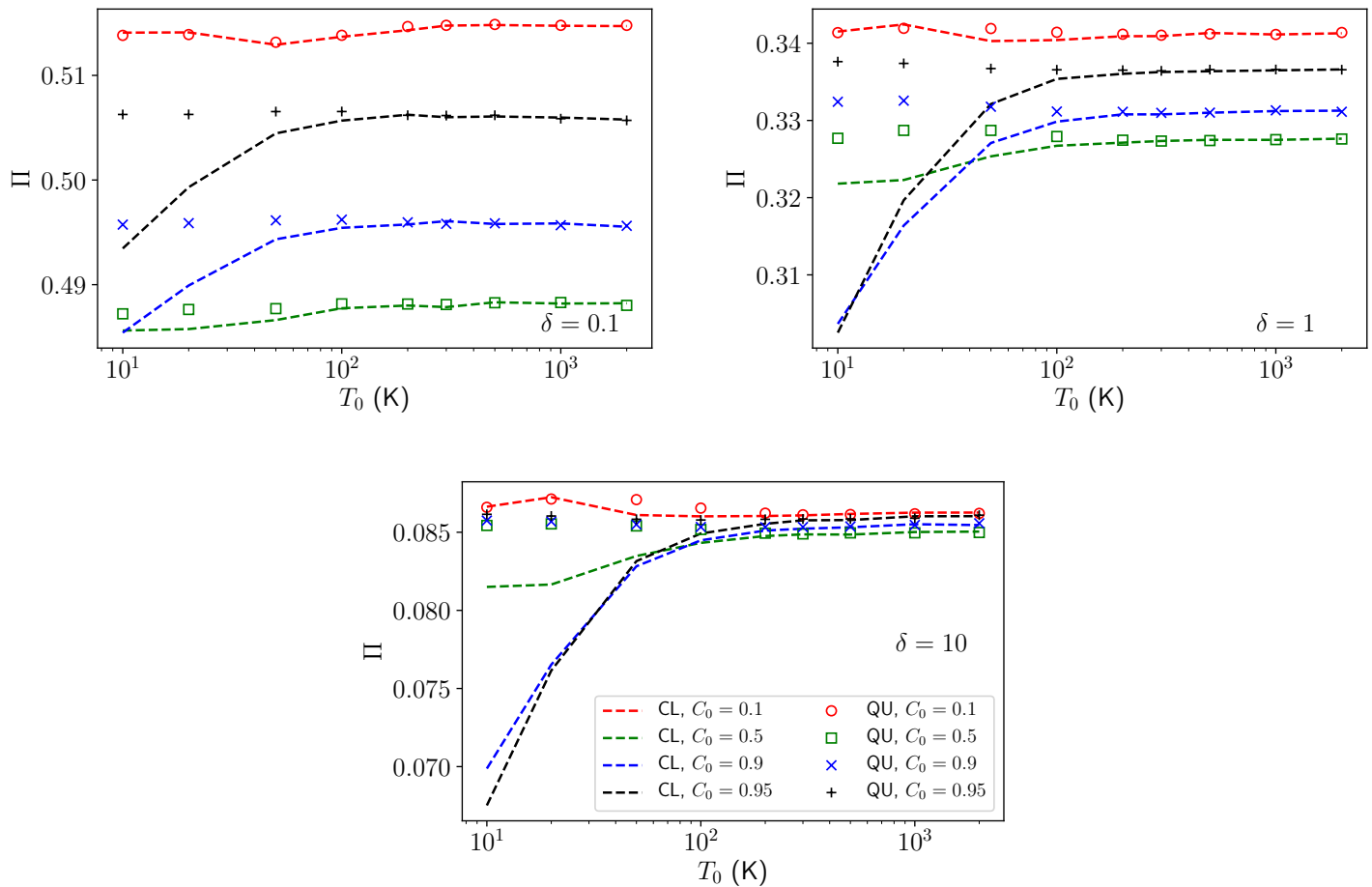

Figure 6: The non-dimensional shear stress $(\Pi)$ as function of the equilibrium temperature $T_{0}$ predicted by the classical and quantum approaches at different rarefaction parameter $(\delta)$ and equilibrium mole fractions $\left(C_{0}\right)$. In each subfigure, the red, green, blue and black lines/symbols represent the results of $C_{0}=0.1,0.5,0.9$, and 0.95 , respectively (color online), in which the lines and symbols correspond to the classical and quantum approaches, respectively. The data in these plots are provided in Table 2 of the Supplementary Material. 

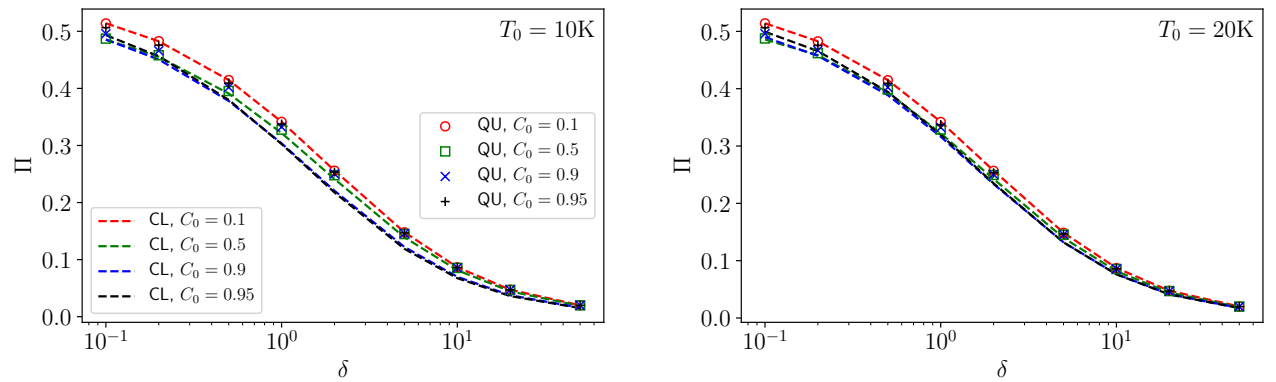

Figure 7: The non-dimensional shear stress ( $\Pi$ ) as functions of $\delta$ for different $C_{0}$ at $T_{0}=10 \mathrm{~K}$ and $20 \mathrm{~K}$ for the Couette flow. The line legends are the same as in Fig. 6 The data in this plot are provided in Table 4 of the Supplementary Material.

\subsubsection{Temperature profiles}

In the Couette flow, the heat generated by the shear flow leads to a non-uniform temperature profile. In the hydrodynamic regime $(\delta \rightarrow \infty)$, the temperature profile is parabolic due to the constant shear rate in the domain. In the free molecular regime $(\delta \rightarrow 0)$, the temperature is a constant i.e., $T=T_{0}\left[1+\left(U_{w} / v_{0}\right)^{2} / 6\right] \approx$ $1.16667 T_{0}$.

Figure 8 shows the mixture temperature profiles at low temperature $\left(T_{0} \leq 20 \mathrm{~K}\right)$ and mole fraction close to unity $\left(C_{0}=0.9\right.$ and 0.95$)$, where the differences between the predictions using the classical and quantum approaches are visually distinguishable. The largest difference appears in the case of $T_{0}=10 \mathrm{~K}, C_{0}=0.95$ and $\delta=1$ as expected. The absolute difference in the temperature profiles increases slightly towards the plates. We note, however, the overall differences in the temperature profiles due to the quantum effects are quite small, as indicated in Table 2, which presents the temperatures at the center of the domain $[T(x=0)]$ for the cases of $T_{0} \leq 100 \mathrm{~K}$. The maximum relative difference of $T(x=0)$ is less than $0.44 \%$. The table also reveals a weak dependence of the non-dimensional temperature profiles on $T_{0}$.

\subsubsection{Velocity profiles}

Similar to the analysis of the temperature profiles above, here we compare the shear velocity profiles predicted by the classical and quantum approaches for low $T_{0}$ and close to unity $C_{0}$ where the differences are observable. Figure 9 shows the non-dimensional velocity profiles. The quantum approach predicts slightly higher slip velocities. To quantify these differences, we extract the non-dimensional velocity gradients, defined as

$$
\nu=\left.\frac{H}{v_{0}} \frac{\mathrm{d} u_{y}}{\mathrm{~d} x}\right|_{x=0},
$$

from the velocity profiles. The gradient $d u_{y} / d x$ is computed from a fitted function in the form of $u_{y}(x)=$ $v_{0}\left[a(x / H)^{3}+b x / H\right]$ from the velocities of the centermost 100 cells. The $\nu$-values are listed in Table 3 . The largest relative difference by the classical and quantum approaches is $18 \%$, appearing in the case of $T_{0}=10$ 

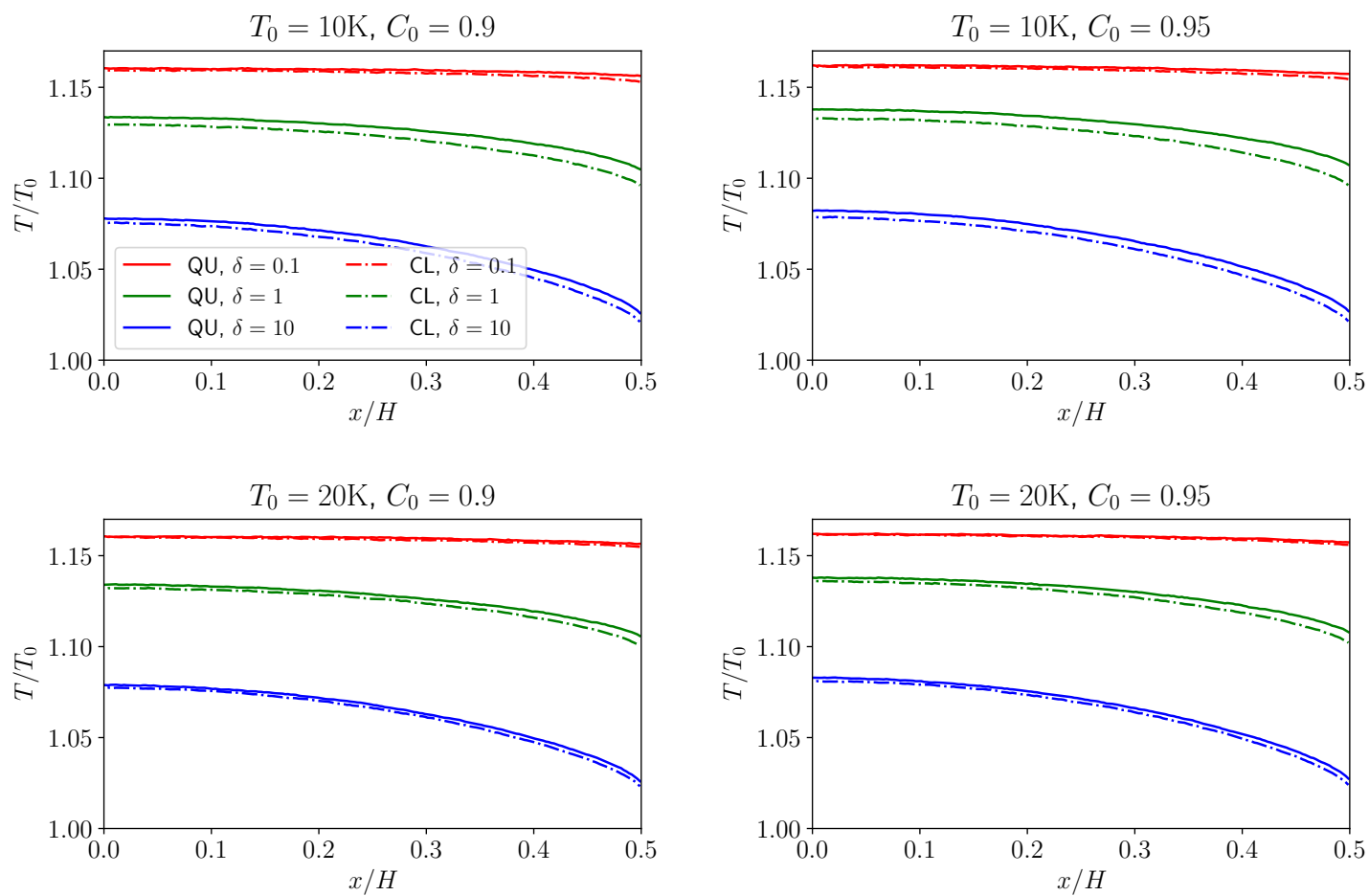

Figure 8: Non-dimensional temperature profiles at different $T_{0}$ and $C_{0}$ for the Couette flow. In each subfigure, the red, green and blue lines are the profiles with $\delta=0.1,1$ and 10, respectively. The solid and dashed lines correspond to the profiles obtained by the quantum- and classical-scattering approaches, respectively

Table 2: Non-dimensional temperatures at the middle point of the domain $(x=0)$, interpolated using the two centermost cells.

\begin{tabular}{|c|c|c|c|c|c|c|c|c|c|}
\hline \multirow[t]{3}{*}{$\delta$} & \multirow[t]{3}{*}{$T_{0}(\mathrm{~K})$} & \multicolumn{8}{|c|}{$T(x=0) / T_{0}$} \\
\hline & & \multicolumn{2}{|c|}{$C_{0}=0.1$} & \multicolumn{2}{|c|}{$C_{0}=0.5$} & \multicolumn{2}{|c|}{$C_{0}=0.9$} & \multicolumn{2}{|c|}{$C_{0}=0.95$} \\
\hline & & CL & $\mathrm{QU}$ & CL & $\mathrm{QU}$ & CL & $\mathrm{QU}$ & CL & $\mathrm{QU}$ \\
\hline \multirow{4}{*}{0.1} & 10 & 1.1633 & 1.1633 & 1.1600 & 1.1603 & 1.1593 & 1.1605 & 1.1612 & 1.1620 \\
\hline & 20 & 1.1639 & 1.1646 & 1.1605 & 1.1610 & 1.1601 & 1.1606 & 1.1614 & 1.1620 \\
\hline & 50 & 1.1639 & 1.1641 & 1.1601 & 1.1601 & 1.1602 & 1.1609 & 1.1616 & 1.1619 \\
\hline & 100 & 1.1635 & 1.1636 & 1.1599 & 1.1599 & 1.1603 & 1.1606 & 1.1619 & 1.1619 \\
\hline \multirow{4}{*}{1} & 10 & 1.1423 & 1.1426 & 1.1307 & 1.1328 & 1.1296 & 1.1336 & 1.1330 & 1.1379 \\
\hline & 20 & 1.1432 & 1.1430 & 1.1312 & 1.1328 & 1.1319 & 1.1340 & 1.1360 & 1.1379 \\
\hline & 50 & 1.1425 & 1.1427 & 1.1313 & 1.1313 & 1.1334 & 1.1340 & 1.1377 & 1.1378 \\
\hline & 100 & 1.1418 & 1.1421 & 1.1305 & 1.1308 & 1.1340 & 1.1337 & 1.1380 & 1.1379 \\
\hline \multirow{4}{*}{10} & 10 & 1.0846 & 1.0852 & 1.0722 & 1.0732 & 1.0754 & 1.0779 & 1.0787 & 1.0821 \\
\hline & 20 & 1.0850 & 1.0851 & 1.0721 & 1.0726 & 1.0774 & 1.0789 & 1.0810 & 1.0829 \\
\hline & 50 & 1.0850 & 1.0851 & 1.0736 & 1.0735 & 1.0793 & 1.0792 & 1.0828 & 1.0830 \\
\hline & 100 & 1.0845 & 1.0849 & 1.0740 & 1.0742 & 1.0797 & 1.0799 & 1.0832 & 1.0830 \\
\hline
\end{tabular}


$\mathrm{K}, C_{0}=0.95, \delta=0.1$

\section{Conclusion}

The rarefied Fourier and Couette flows of helium-neon mixture have been simulated by the DSMC method based on the $a b$ initio potentials. Both the classical and quantum approaches to the modeling of inter-atomic scatterings have been applied. The quantum approach allows us to accurately simulate the flows of light-weight gases at the temperature as low as $5 \mathrm{~K}$ where the quantum effects are non-negligible.

The results show that the quantum effects on the heat flux of the Fourier flow and the shear stress of the Couette flow are non-negligible when the temperature is lower than $500 \mathrm{~K}$ and is significant when the temperature is lower than $50 \mathrm{~K}$. The quantum effects are stronger with lower temperature and higher He mole fraction. Without considering the quantum effects, the errors can be as high as $20 \%$ in the prediction of heat flux and $27 \%$ in the shear stress when the equilibrium temperature is $10 \mathrm{~K}$.

The quantum effects have a dramatic effect on the mole fraction distribution of the Fourier flow when the temperature is as low as $20 \mathrm{~K}$, where the mole fraction gradient changes its direction. In comparison, the classical scattering-based approach fails to capture this behavior. The quantum effects on other macroscopic variables such as the temperature and velocity are generally weak and detectable only for He-dominated mixture when the temperature is $20 \mathrm{~K}$ or lower.

In addition, the $a b$ initio potential has been implemented as a collision sub-model in the open source DSMC package of the dsmcFoam+ solver, which is provided in the Supplemental Material.

Table 3: Non-dimensional gradient of the velocity $\nu$ [see Eq. 19] at the center of the domain.

\begin{tabular}{|c|c|c|c|c|c|c|c|c|c|}
\hline \multirow[t]{3}{*}{$\delta$} & \multirow[t]{3}{*}{$T_{0}(\mathrm{~K})$} & \multicolumn{8}{|c|}{$\nu$} \\
\hline & & \multicolumn{2}{|c|}{$C_{0}=0.1$} & \multicolumn{2}{|c|}{$C_{0}=0.5$} & \multicolumn{2}{|c|}{$C_{0}=0.9$} & \multicolumn{2}{|c|}{$C_{0}=0.95$} \\
\hline & & $\mathrm{CL}$ & $\mathrm{QU}$ & $\mathrm{CL}$ & $\mathrm{QU}$ & $\mathrm{CL}$ & QU & $\mathrm{CL}$ & $\mathrm{QU}$ \\
\hline \multirow{4}{*}{0.1} & 10 & 0.1600 & 0.1572 & 0.1640 & 0.1654 & 0.1850 & 0.1622 & 0.1874 & 0.1593 \\
\hline & 20 & 0.1609 & 0.1579 & 0.1679 & 0.1670 & 0.1757 & 0.1588 & 0.1768 & 0.1572 \\
\hline & 50 & 0.1644 & 0.1612 & 0.1617 & 0.1583 & 0.1586 & 0.1614 & 0.1623 & 0.1567 \\
\hline & 100 & 0.1638 & 0.1588 & 0.1576 & 0.1546 & 0.1590 & 0.1535 & 0.1566 & 0.1525 \\
\hline \multirow{4}{*}{1} & 10 & 0.4468 & 0.4495 & 0.4486 & 0.4402 & 0.4800 & 0.4425 & 0.4937 & 0.4467 \\
\hline & 20 & 0.4457 & 0.4447 & 0.4528 & 0.4405 & 0.4625 & 0.4411 & 0.4696 & 0.4431 \\
\hline & 50 & 0.4471 & 0.4487 & 0.4421 & 0.4402 & 0.4425 & 0.4362 & 0.4520 & 0.4439 \\
\hline & 100 & 0.4485 & 0.4475 & 0.4400 & 0.4360 & 0.4387 & 0.4380 & 0.4428 & 0.4417 \\
\hline \multirow{4}{*}{10} & 10 & 0.8265 & 0.8261 & 0.8265 & 0.8191 & 0.8502 & 0.8246 & 0.8552 & 0.8291 \\
\hline & 20 & 0.8252 & 0.8229 & 0.8252 & 0.8184 & 0.8387 & 0.8254 & 0.8452 & 0.8281 \\
\hline & 50 & 0.8229 & 0.8280 & 0.8238 & 0.8204 & 0.8264 & 0.8222 & 0.8294 & 0.8291 \\
\hline & 100 & 0.8297 & 0.8292 & 0.8238 & 0.8222 & 0.8272 & 0.8291 & 0.8291 & 0.8341 \\
\hline
\end{tabular}



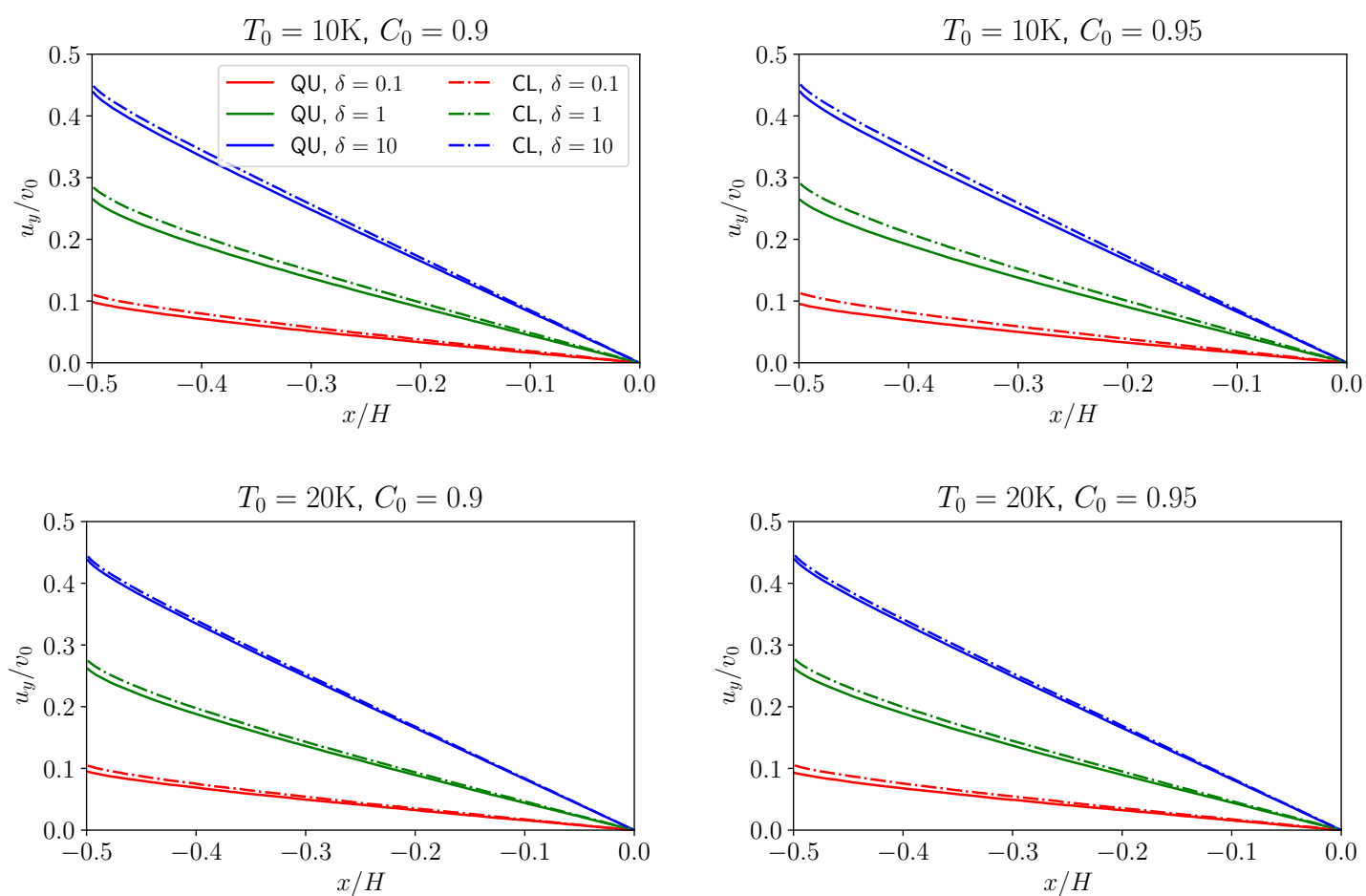

Figure 9: Non-dimensional velocity profiles at different $T_{0}$ and $C_{0}$ for the Couette flow. In each subfigure, the red, green and blue lines are the profiles with $\delta=0.1,1$ and 10, respectively. The solid and dashed lines represent the quantum- and classical-scattering approaches, respectively. 


\section{Supplementary material}

The collision procedure based on the ab initio potentials is implemented as a collision sub-model for the dsmcFoam+ solver. The source code and the deflection angles matrices are hosted on GitHub and can be freely downloaded at https://github.com/zhulianhua/AbInitio. All the solutions reported in this article can be reproduced with this sub-model in the dsmcFoam + solver. The matrix of the deflection angle cosine for He-Ne collisions is also given in the supplementary material of the present paper in the file "xiHe4-Ne.csv". Sec. 3.1 explains how to use it. The numerical data, including the temperature, velocity, mole fraction distributions, and wall shear stress/heat flux for all the cases, are provided in the supplementary material and can be obtained under https://doi.org/10.15129/fdd139b7-e6a2-4388-9b54-b61283ae5b5e

\section{Acknowledgement}

The project leading to this paper has received funding from the European Union's Horizon 2020 Research and Innovation Programme under the Marie Skłodowska-Curie grant agreement number 793007. F. Sharipov acknowledges the Brazilian Agency CNPq, Brazil for the support of his research, grant 304831/2018-2. L. Wu acknowledges the National Natural Science Foundation of China under the grant No. 11872024. Financial support from the UK Engineering and Physical Sciences Research Council (EPSRC) under grant No. EP/M021475/1 is gratefully acknowledged. Computing time during the program development \& testing on the ARCHER is provided by the "UK Consortium on Mesoscale Engineering Sciences (UKCOMES)" under the UK EPSRC grant Nos. EP/L00030X/1 and EP/R029598/1. Initial testing simulations have also run on the EPSRC funded ARCHIE-WeSt High Performance Computer (EPSRC Grant No. EP/K000586/1).

\section{References}

[1] A. Hart, N. Gnanendran, Cryogenic CO2 capture in natural gas, Energy Procedia 1 (2009) 697-706. doi:10.1016/j. egypro.2009.01.092

[2] H. Oh, M. Hirscher, Quantum sieving for separation of Hydrogen isotopes using MOFs, European Journal of Inorganic Chemistry 2016 (2016) 4278-4289. doi:10.1002/ejic.201600253.

[3] S. A. Fitzgerald, K. Shinbrough, K. H. Rigdon, J. L. C. Rowsell, M. T. Kapelewski, S. H. Pang, K. V. Lawler, P. M. Forster, Temperature-programmed desorption for isotope separation in nanoporous materials, The Journal of Physical Chemistry C 122 (2018) 1995-2001. doi:10.1021/acs.jpcc.7b11048

[4] V. Kalinin, E. Tada, F. Millet, N. Shatil, ITER cryogenic system, Fusion Engineering and Design 81 (2006) $2589-2595$. doi:10.1016/j.fusengdes.2006.07.003

[5] L. Graber, W. J. Kim, P. Cheetham, C. H. Kim, H. Rodrigo, S. V. Pamidi, Dielectric properties of cryogenic gas mixtures containing helium, neon, and hydrogen, IOP Conference Series: Materials Science and Engineering 102 (2015) 012018. doi:10.1088/1757-899X/102/1/012018

[6] P. Cheetham, W. Kim, C. H. Kim, L. Graber, H. Rodrigo, S. Pamidi, Enhancement of dielectric strength of cryogenic gaseous helium by addition of small mol\% hydrogen, IEEE Transactions on Applied Superconductivity 27 (2017) 1-5. doi:10.1109/TASC.2016.2642539

[7] S. Varoutis, C. Day, Numerical modeling of an ITER type Cryopump, Fusion Engineering and Design 87 (2012) $1395-1398$. doi:10.1016/j.fusengdes.2012.03.023

[8] K. Jousten (Ed.), Handbook of Vacuum Technology, 2nd Edition, WILEY-VCH, Weinheim, 2016.

[9] G. A. Bird, Molecular Gas Dynamics and the Direct Simulation of Gas Flows, Clarendon Press, Oxford, 1994.

[10] G. A. Bird, The DSMC Method, CreateSpace Independent Publishing Platform, 2013.

[11] F. Sharipov, Rarefied Gas Dynamics: Fundamentals for Research and Practice, John Wiley \& Sons, Weinheim, 2015.

[12] S. M. Cybulski, R. R. Toczyłowski, Ground state potential energy curves for He2, Ne2, Ar2, He-Ne, He-Ar, and Ne-Ar: A coupled-cluster study, The Journal of Chemical Physics 111 (1999) 10520-10528. doi:10.1063/1.480430 
[13] M. Przybytek, W. Cencek, J. Komasa, G. Łach, B. Jeziorski, K. Szalewicz, Relativistic and Quantum Electrodynamics Effects in the Helium Pair Potential, Physical Review Letters 104 (2010) 183003. doi:10.1103/PhysRevLett.104.183003

[14] R. Hellmann, E. Bich, E. Vogel, Ab initio potential energy curve for the neon atom pair and thermophysical properties of the dilute neon gas. I. Neon-neon interatomic potential and rovibrational spectra, Molecular Physics 106 (2008) $133-140$. doi:10.1080/00268970701843147

[15] J. López Cacheiro, B. Fernández, D. Marchesan, S. Coriani, C. Hättig, A. Rizzo, Coupled cluster calculations of the ground state potential and interaction induced electric properties of the mixed dimers of helium, neon and argon, Molecular Physics 102 (2004) 101-110. doi:10.1080/00268970410001668606

[16] F. Sharipov, J. L. Strapasson, Direct simulation Monte Carlo method for an arbitrary intermolecular potential, Physics of Fluids 24 (2012) 011703. doi:10.1063/1.3676060

[17] F. Sharipov, J. L. Strapasson, Ab initio simulation of transport phenomena in rarefied gases, Physical Review E 86 (2012) 031130. doi:10.1103/PhysRevE.86.031130

[18] F. Sharipov, J. L. Strapasson, Benchmark problems for mixtures of rarefied gases. I. Couette flow, Physics of Fluids 25 (2013) 027101. doi:10.1063/1.4791604

[19] F. Sharipov, V. J. Benites, Transport coefficients of helium-neon mixtures at low density computed from ab initio potentials, The Journal of Chemical Physics 147 (2017) 224302. doi:10.1063/1.5001711

[20] F. Sharipov, Modeling of transport phenomena in gases based on quantum scattering, Physica A: Statistical Mechanics and its Applications 508 (2018) 797-805. doi:10.1016/j.physa.2018.05.129

[21] F. Sharipov, Influence of quantum intermolecular interaction on internal flows of rarefied gases, Vacuum 156 (2018) 146-153. doi:10.1016/j.vacuum.2018.07.022

[22] F. Sharipov, F. C. Dias, Temperature dependence of shock wave structure in helium and neon, Physics of Fluids 31 (2019) 037109. doi:10.1063/1.5088556.

[23] E. P. Gross, S. Ziering, Heat flow between parallel plates, The Physics of Fluids 2 (1959) 701-712. doi:10.1063/1.1705975

[24] M. L. Lavin, J. K. Haviland, Application of a moment method to heat transfer in rarefied gases, The Physics of Fluids 5 (1962) 274-279. doi:10.1063/1.1706611

[25] K. Frankowski, Z. Alterman, C. L. Pekeris, Heat transport between parallel plates in a rarefied gas of rigid sphere molecules, The Physics of Fluids 8 (1965) 245-258. doi:10.1063/1.1761217

[26] P. Bassanini, C. Cercignani, C. D. Pagani, Comparison of kinetic theory analyses of linearized heat transfer between parallel plates, International Journal of Heat and Mass Transfer 10 (1967) 447-460. doi:10.1016/0017-9310(67) 90165-2

[27] S. K. Hsu, T. F. Morse, Kinetic theory of parallel plate heat transfer in a polyatomic gas, The Physics of Fluids 15 (1972) 584-591. doi:10.1063/1.1693952

[28] J. R. Thomas, T. S. Chang, C. E. Siewert, Heat transfer between parallel plates with arbitrary surface accommodation, The Physics of Fluids 16 (1973) 2116-2120. doi:10.1063/1.1694275

[29] I. A. Graur, A. P. Polikarpov, Comparison of different kinetic models for the heat transfer problem, Heat and Mass Transfer 46 (2009) 237-244. doi:10.1007/s00231-009-0558-x

[30] L. Wu, H. Liu, Y. Zhang, J. M. Reese, Influence of intermolecular potentials on rarefied gas flows: Fast spectral solutions of the Boltzmann equation, Physics of Fluids 27 (2015) 082002. doi:doi/full/10.1063/1.4929485

[31] D. Valougeorgis, Couette flow of a binary gas mixture, The Physics of Fluids 31 (1988) 521-524. doi:10.1063/1.866833

[32] C. E. Siewert, Couette flow for a binary gas mixture, Journal of Quantitative Spectroscopy and Radiative Transfer 70 (2001) 321-332. doi:10.1016/S0022-4073(00)00143-6.

[33] F. Sharipov, L. M. Gramani Cumin, D. Kalempa, Plane Couette flow of binary gaseous mixture in the whole range of the Knudsen number, European Journal of Mechanics - B/Fluids 23 (2004) 899-906. doi:10.1016/j.euromechflu.2004.03. 002 .

[34] R. D. M. Garcia, C. E. Siewert, The McCormack model for gas mixtures: Heat transfer in a plane channel, Physics of Fluids 16 (2004) 3393-3402. doi:10.1063/1.1773711

[35] R. D. M. Garcia, C. E. Siewert, The McCormack model for gas mixtures: Plane Couette flow, Physics of Fluids 17 (2005) 037102. doi:10.1063/1.1845911.

[36] F. Sharipov, L. M. G. Cumin, D. Kalempa, Heat flux between parallel plates through a binary gaseous mixture over the whole range of the Knudsen number, Physica A: Statistical Mechanics and its Applications 378 (2007) $183-193$. doi:10.1016/j.physa.2006.11.077

[37] M. T. Ho, L. Wu, I. Graur, Y. Zhang, J. M. Reese, Comparative study of the Boltzmann and McCormack equations for Couette and Fourier flows of binary gaseous mixtures, International Journal of Heat and Mass Transfer 96 (2016) $29-41$. doi:10.1016/j.ijheatmasstransfer.2015.12.068

[38] C. Tantos, D. Valougeorgis, Conductive heat transfer in rarefied binary gas mixtures confined between parallel plates based on kinetic modeling, International Journal of Heat and Mass Transfer 117 (2018) 846-860. doi:10.1016/j. ijheatmasstransfer.2017.10.050

[39] Y. Zhang, L. Zhu, P. Wang, Z. Guo, Discrete unified gas kinetic scheme for flows of binary gas mixture based on the McCormack model, Physics of Fluids 31 (2019) 017101. doi:10.1063/1.5063846

[40] F. J. McCormack, Construction of linearized kinetic models for gaseous mixtures and molecular gases, The Physics of Fluids 16 (1973) 2095-2105. doi:10.1063/1.1694272

[41] S. Kosuge, Model Boltzmann equation for gas mixtures: Construction and numerical comparison, European Journal of Mechanics - B/Fluids 28 (2009) 170-184. doi:10.1016/j.euromechflu.2008.05.001

[42] S. Kosuge, K. Aoki, S. Takata, Heat transfer in a gas mixture between two parallel plates: Finite-difference analysis of the Boltzmann equation, AIP Conference Proceedings 585 (2001) 289-296. doi:10.1063/1.1407574

[43] J. L. Strapasson, F. Sharipov, Ab initio simulation of heat transfer through a mixture of rarefied gases, International 
Journal of Heat and Mass Transfer 71 (2014) 91-97. doi:10.1016/j.ijheatmasstransfer.2013.12.011

[44] H. Yamaguchi, J. Hosoi, Y. Matsuda, T. Niimi, Measurement of conductive heat transfer through rarefied binary gas mixtures, Vacuum 160 (2019) 164-170. doi:10.1016/j.vacuum.2018.11.021

[45] H. Schlichting, D. Menzel, T. Brunner, W. Brenig, J. C. Tully, Quantum effects in the sticking of ne on a flat metal surface, Phys. Rev. Lett. 60 (1988) 2515-2518. doi:10.1103/PhysRevLett.60.2515. URL https://link.aps.org/doi/10.1103/PhysRevLett.60.2515

[46] W. Wagner, A convergence proof for Bird's direct simulation Monte Carlo method for the Boltzmann equation, Journal of Statistical Physics 66 (1992) 1011-1044. doi:10.1007/BF01055714

[47] C. White, M. K. Borg, T. J. Scanlon, S. M. Longshaw, B. John, D. R. Emerson, J. M. Reese, dsmcFoam+: An OpenFOAM based direct simulation Monte Carlo solver, Computer Physics Communications 224 (2018) 22-43. doi:10.1016/j.cpc. 2017.09 .030

[48] F. Sharipov, V. J. Benites, Transport coefficients of helium-argon mixture based on ab initio potential, The Journal of Chemical Physics 143 (2015) 154104. doi:10.1063/1.4933327

[49] L. Wu, C. White, T. J. Scanlon, J. M. Reese, Y. H. Zhang, Deterministic numerical solutions of the Boltzmann equation using the fast spectral method, Journal of Computational Physics 250 (2013) 27-52. doi:DOI10.1016/j.jcp. 2013.05.003

[50] T. I. Moran, W. W. Watson, Thermal diffusion factors for the noble gases, Physical Review 109 (1958) 1184-1190. doi:10.1103/PhysRev.109.1184 